\title{
Evaluación de indicadores biológicos en suelos de la región semiárida pampeana central
}

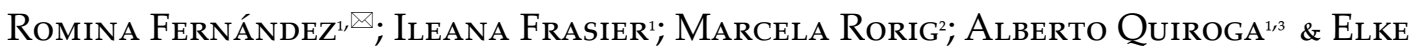 \\ NOELLEMEYER $^{3}$
}

${ }^{1}$ INTA Anguil. La Pampa, Argentina. ${ }^{2}$ CIRN Suelos, INTA Castelar. Buenos Aires, Argentina. ${ }^{3}$ Universidad Nacional de La Pampa. La Pampa, Argentina.

\begin{abstract}
RESUMEN. En la región semiárida pampeana aún no se han usado indicadores biológicos para valorar cambios en la calidad del suelo relacionados con el uso de la tierra. El objetivo de este trabajo fue caracterizar, evaluar y obtener valores de línea de base de respiración de suelo, biomasa microbiana y cociente metabólico en suelos de la región semiárida pampeana bajo vegetación natural y agricultura. El estudio se desarrolló en Paleustoles petrocálcicos de la planicie con tosca de la región semiárida pampeana central. Determinamos los contenidos de arcilla y limo, la porosidad total, el contenido de carbono y nitrógeno, la respiración y la biomasa microbiana, la relación hongo/bacteria y el cociente metabólico en los estratos superior, medio e inferior de dos suelos con vegetación natural y otros dos bajo uso agrícola. El tipo de uso del suelo influyó significativamente sobre la actividad y el tamaño de la biota, de manera tal que los suelos agrícolas, con menores contenidos de carbono y nitrógeno, tuvieron una menor respiración y menores contenidos de carbono y nitrógeno en la biomasa microbiana, en relación con los suelos bajo vegetación natural. El cociente metabólico no presentó diferencias entre suelos bajo distinto uso, por lo que no fue de utilidad para indicar el estado de degradación edáfico, en comparación con la biomasa microbiana y la respiración. El uso del suelo también afectó la relación hongo/bacteria, ya que la participación de hongos en la biomasa microbiana de los suelos bajo uso agrícola fue considerablemente menor que la de los suelos bajo vegetación natural. Estos resultados nos permitieron establecer valores umbrales y de línea de base para la respiración del suelo y el carbono de la biomasa microbiana.
\end{abstract}

[Palabras clave: respiración de suelo, biomasa microbiana, relación hongo/bacteria, cociente metabólico]

\begin{abstract}
Evaluation of biological indicators in soils of the central semiarid Pampa region. In the semiarid Pampa, biological indicators have not yet been used to evaluate the effect of land-use on soil quality. Our objective was to characterize, evaluate and obtain base-line values for soil respiration, microbial biomass and metabolic quotient in agricultural and native vegetation soils of the central semiarid Pampa region. In two natural vegetation and two agricultural soils, we determined clay, silt, total porosity, total carbon and nitrogen contents, microbial respiration and biomass, fungi-bacteria ratio and metabolic quotient in the upper, middle and lower profile layers. Soil use significantly affected the activity and the size of soil biota, in the sense that the agricultural soils, with lower carbon and nitrogen contents, showed lower respiration and microbial biomass carbon and nitrogen contents compared to the natural vegetation. Microbial biomass and respiration were better indicators for soil degradation than the metabolic quotient. Soil use also had an effect on the fungi/bacteria ratio since agricultural soils had a lower proportion of fungi than soils under natural vegetation. These results enable us to establish baseline and threshold values for respiration and microbial biomass carbon contents.
\end{abstract}

[Keywords: soil respiration, microbial biomass, fungi/bacteria ratio, metabolic quotient]

\section{INTRODUCCIÓN}

Los microorganismos tienen influencia en diversos procesos y funciones de los ecosistemas naturales y agrícolas (Ponge 2015). Los parámetros biológicos y bioquímicos tienden a reaccionar de manera rápida y son sensibles a los cambios producidos por el manejo, por lo que pueden constituir una señal temprana para estimar la calidad edáfica, incluso antes que las propiedades físicas y químicas (Nannipieri 1994; Romaniuk et al. 2016). En este sentido, numerosas investigaciones sugieren el uso de indicadores que reflejen la actividad, la abundancia y la

Editora asociada: María Semmartin

fernandez.romina@inta.gob.ar diversidad de los microorganismos edáficos (Moscatelli et al. 2007).

A pesar de que existe un consenso general acerca de que los parámetros biológicos resultan necesarios para describir el efecto de cambios en el uso del suelo sobre las funciones ecosistémicas (van Bruggen and Semenov 2000; Figuerola et al. 2012). Benayas y colaboradores (2017) concluyeron que la relación entre la biodiversidad y la provisión de servicios ecosistémicos es incierta y que aún no existe un conjunto de métodos y de variables que permitan determinar la calidad biológica de suelos (Philippot et al. 2012).

Recibido: 28 de marzo de 2017

Aceptado: 29 de diciembre de 2017 
En una iniciativa reciente, un análisis de transectas continentales buscó establecer los valores de referencia de diferentes variables que se pueden considerar como indicadores (Griffiths et al. 2016).

Cuatro indicadores de uso frecuente son la respiración microbiana, el carbono de la biomasa microbiana, el cociente metabólico (que relaciona los dos anteriores) y la relación hongo/bacteria. La respiración microbiana, evaluada a través de la producción de $\mathrm{CO}_{2}$, refleja directamente la actividad microbiana e indirectamente la disponibilidad de sustrato (Noellemeyer et al. 2008; Fernández et al. 2010). En general, una mayor respiración se asocia a un incremento en la actividad microbiana; sin embargo, las tasas de respiración por encima de un valor límite podrían representar una pérdida excesiva de carbono para el ecosistema (Cotrufo et al. 2013; Geyer et al. 2016). Se propuso a la respiración basal como un indicador de calidad biológica; es el valor de respiración a tasa constante de un determinado suelo y caracteriza la actividad microbiana en condiciones de equilibrio (Ashman 2003; Ashman et al. 2009; Wang and Post 2012). En el caso del carbono de la biomasa microbiana, parte de su relevancia radica en que si bien constituye una pequeña fracción del carbono total, es un reservorio de nutrientes para las plantas (Kallenbach et al. 2016) aun más lábil que la materia orgánica (Costantini et al. 1996). Se ha demostrado que tanto la actividad como la composición de la comunidad microbiana del suelo son susceptibles a cambios asociados a las prácticas de manejo como el sistema de labranza y la rotación de cultivos (Bossio et al. 2005). Sin embargo, se plantea que tanto la respiración como el carbono de la biomasa microbiana por sí solos no reflejarían el estado de salud edáfico ya que un suelo con alto contenido de carbono microbiano no necesariamente respira más y un suelo con mayor tasa de respiración no siempre presenta mayor carbono microbiano (Anderson 2003). La relación entre la respiración basal y la biomasa microbiana, conocida como el cociente metabólico $\left(\mathrm{qCO}_{2}\right)$ (Anderson and Domsch 1990), brinda una interpretación más directa de la actividad de los microorganismos en relación a su biomasa (Pascaud et al. 2012). Se lo considera un indicador útil del disturbio del ecosistema: valores superiores reflejan mayor eficiencia microbiana en el uso energético y su valor disminuye con la madurez del ecosistema (Odum 1969; Anderson and Domsch 1990;
Anderson and Domsch 1993). Sin embargo, un meta-análisis concluyó que la respuesta del cociente metabólico a los disturbios o al estrés en el ecosistema puede ser muy poco predecible (Wardle and Ghani 1995). Finalmente, la relación hongo/bacteria es un parámetro que se considera indicativo de la respuesta del suelo al cambio ambiental. No obstante, no siempre se cumplen las expectativas en cuanto a la dirección del cambio en este indicador como resultado de un disturbio o una modificación en el manejo del suelo (Strickland and Rousk 2010), y existen factores edáficos que pueden alterar esta relación (e.g., el pH [Fierer et al. 2005] y la profundidad en el perfil [Sun et al. 2011]).

A lo largo del tiempo, el uso del suelo ha producido cambios en el ambiente edáfico (Benayas et al. 2017), lo que incide sobre las propiedades biológicas y las condiciones físicas. Esto, a su vez, afecta el hábitat donde los microorganismos se desarrollan y se reproducen (Kravchenko et al. 2014; Negassa et al. 2015). Segnestam (2002) señaló la importancia de a) establecer la línea de base de una actividad que puede impactar de manera positiva o negativa sobre el ambiente, $b$ ) determinar umbrales para controlar el seguimiento de impactos negativos que no deben exceder un nivel predeterminado, y c) fijar objetivos o metas que permitan evaluar el impacto de una respuesta. Los valores de línea de base expresan un valor de referencia de un determinado indicador (Micó et al. 2007); se suelen obtener en suelos que corresponden a un estado deseable de salud o de calidad. En muchos casos, estos serían suelos bajo vegetación prístina, pero también se utilizan aquellos que se encuentren poco disturbados cuando no existen situaciones prístinas (Thackway and Specht 2015). En este contexto, los valores de línea de base resultarían útiles para interpretar los niveles actuales de las variables indicadoras y para evaluar el efecto de las prácticas de uso de los suelos.

En la región semiárida pampeana, las investigaciones acerca de la cuantificación de los indicadores biológicos que reflejen cambios en el uso del suelo son incipientes y no existen valores de línea de base o de referencia. El objetivo de este trabajo fue caracterizar, evaluar y obtener valores de línea de base, de respiración de suelo, de biomasa microbiana y del cociente metabólico en suelos agrícolas y bajo vegetación natural de la región semiárida pampeana. Se planteó como hipótesis que la respiración de suelo, la biomasa microbiana y 
el cociente metabólico de suelos en vegetación natural podrían ser considerados valores de línea de base.

\section{Materiales y Métodos}

\section{Sitios de estudio y diseño experimental}

El estudio se desarrolló sobre Paleustoles petrocálcicos ubicados en el sur de la planicie con tosca de la región semiárida pampeana (Figura 1). Se seleccionaron 4 suelos por diferencias en las prácticas de producción, ubicados en un radio de $40 \mathrm{~km}$ de distancia, entre los $36^{\circ} 25^{\prime} 1,38^{\prime \prime} \mathrm{S}, 64^{\circ} 3^{\prime} 14^{\prime \prime} \mathrm{O}$ y los $36^{\circ}$ $33^{\prime} 34,82^{\prime \prime} \mathrm{S}, 64^{\circ} 16^{\prime} 47,86^{\prime \prime} \mathrm{O}$. Dos de ellos presentan pastizales naturales con vegetación dominante de gramíneas perennes por más de 50 años, con contenidos de carbono orgánico y nitrógeno diferentes entre sí y superiores a dos suelos seleccionados bajo uso agrícola también por más de 50 años (Tabla 1). Las especies dominantes del pastizal natural eran Stipa tenuis, Poa ligularis, Bromus brevis y Piptochaetium napostaense. Estos ambientes se utilizan para pastoreo de ganado vacuno y se encuentran en potreros de $\sim 50$ ha. En uno de los sitios (sitio 2) también se registró la presencia de especies arbóreas leguminosas. Los dos suelos bajo uso agrícola fueron cultivados en siembra directa no continua que alternó con labranza de discos. Los cultivos más frecuentes fueron trigo (Triticum aestivum), girasol (Helianthus annuus), maíz (Zea mays), sorgo (Sorghum bicolor) y cebada (Hordeum vulgare).
En cada suelo se muestrearon tres perfiles equidistantes $10 \mathrm{~m}$ cada uno. En cada perfil se consideraron tres estratos no continuos en cuanto a la profundidad: 1) estrato superior, de 0 a $6 \mathrm{~cm}$ de profundidad, relacionado con la captación del agua de las precipitaciones, 2) estrato medio, entre los 17 y los $23 \mathrm{~cm}$ de profundidad, donde mayormente ocurren las densificaciones de suelo, y 3) estrato inferior, entre los 40 y los $46 \mathrm{~cm}$ de profundidad, que representaría las características del material parental, ya que corresponde al horizonte C. En cada uso de suelo y en los tres perfiles se extrajeron muestras de los tres estratos; en el suelo seco al aire y tamizado por $2 \mathrm{~mm}$ se determinaron las siguientes variables: a) contenido de arcilla y limo mediante el método de la pipeta de Robinson y contenido de las fracciones de arena por tamizado (en cada perfil de suelo se tomó una muestra compuesta de 3 submuestras por estrato), y b) contenido de carbono y nitrógeno total por combustión seca (LECO Truspec). El contenido de carbono obtenido mediante ésta técnica fue considerado carbono orgánico, ya que los suelos no presentaron carbonato de calcio libre. En cada perfil de suelo se tomaron 2 muestras compuestas de 3 submuestras por cada estrato de profundidad (Tabla 1).

\section{Determinaciones biológicas}

Respiración del suelo. A fin de determinar respiración de suelo, en cada uso de suelo y en los tres perfiles se extrajeron 3 cilindros de $113 \mathrm{~cm}^{3}$ por estrato de profundidad.

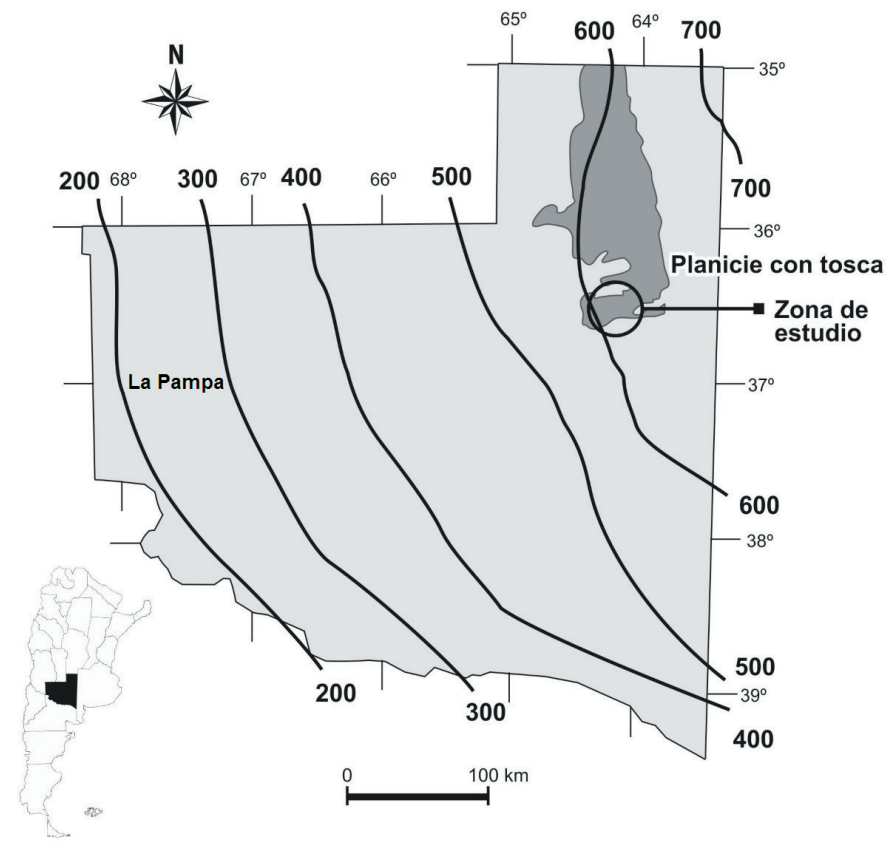

Figura 1. Mapa de Argentina y de la provincia de La Pampa, donde se detalla la zona de estudio. Se indica la planicie con tosca y las isohietas.

Figure 1. Map of Argentina and La Pampa, with a detailed map of the study area. The tosca plain area and the corresponding isohyets are indicated. 
Tabla 1. Propiedades físicas y químicas de los suelos bajo diferentes usos: vegetación natural (VN1 y VN2) y agricultura (A1 y A2). Letras distintas indican diferencias significativas entre usos para el mismo estrato.

Table 1. Physical and chemical properties of soils under different use in natural vegetation (VN1 and VN2) and agriculture (A1 and A2). Different letters indicate significant differences between soil uses for the same depth layer.

\begin{tabular}{|c|c|c|c|c|}
\hline & \multicolumn{2}{|c|}{ Vegetación natural } & \multicolumn{2}{|c|}{ Agricultura } \\
\hline & VN1 & VN2 & A1 & A2 \\
\hline & \multicolumn{4}{|c|}{ Secuencia de horizontes } \\
\hline Superior & $\mathrm{A}_{1} / \mathrm{A}_{2}$ & A & A & A \\
\hline Medio & $\mathrm{AC}$ & $\mathrm{AC}$ & $\mathrm{AC}$ & $\mathrm{AC}$ \\
\hline \multirow[t]{2}{*}{ Inferior } & $\mathrm{C}-\mathrm{Ckm}$ & $\mathrm{C}-\mathrm{Ckm}$ & $\mathrm{C}-\mathrm{Ckm}$ & $\mathrm{C}-\mathrm{Ckm}$ \\
\hline & \multicolumn{4}{|c|}{ Clase textural } \\
\hline Superior & Franco & Franco & Franco arenoso & Franco \\
\hline Medio & Franco & Franco arenoso & Franco arenoso & Franco \\
\hline \multirow[t]{2}{*}{ Inferior } & Franco arenoso & Franco arenoso & Franco arenoso & Franco \\
\hline & \multicolumn{4}{|c|}{ Carbono orgánico (\%) } \\
\hline Superior & $2.5 \mathrm{~B}$ & $6.9 \mathrm{~A}$ & $1.0 \mathrm{C}$ & $1.4 \mathrm{C}$ \\
\hline Medio & $1.0 \mathrm{~A}$ & $0.7 \mathrm{~B}$ & $0.6 \mathrm{D}$ & $0.9 \mathrm{C}$ \\
\hline \multirow[t]{2}{*}{ Inferior } & $11.0 \mathrm{~A}$ & $0.4 \mathrm{C}$ & $0.4 \mathrm{C}$ & $0.7 \mathrm{~B}$ \\
\hline & \multicolumn{4}{|c|}{ Nitrógeno orgánico (\%) } \\
\hline Superior & $0.17 \mathrm{~B}$ & $0.74 \mathrm{~A}$ & $0.01 \mathrm{C}$ & $0.14 \mathrm{BC}$ \\
\hline Medio & $0.09 \mathrm{~A}$ & $0.09 \mathrm{~A}$ & $0.07 \mathrm{~B}$ & $0.08 \mathrm{~A}$ \\
\hline \multirow[t]{2}{*}{ Inferior } & $0.07 \mathrm{~A}$ & $0.04 \mathrm{~B}$ & $0.05 \mathrm{~B}$ & $0.06 \mathrm{~A}$ \\
\hline & \multicolumn{4}{|c|}{ Porosidad total (\%) } \\
\hline Superior & $58 \mathrm{~B}$ & $68 \mathrm{~A}$ & $33 \mathrm{D}$ & $46 \mathrm{C}$ \\
\hline Medio & $58 \mathrm{~A}$ & $56 \mathrm{~A}$ & $36 \mathrm{~B}$ & $39 \mathrm{~B}$ \\
\hline \multirow[t]{2}{*}{ Inferior } & $53 \mathrm{~A}$ & $48 \mathrm{BC}$ & $42 \mathrm{C}$ & $50 \mathrm{~B}$ \\
\hline & \multicolumn{4}{|c|}{ Densidad aparente $\left(\mathrm{g} / \mathrm{cm}^{3}\right)$} \\
\hline Superior & $1.0 \mathrm{C}$ & $0.85 \mathrm{D}$ & $1.37 \mathrm{~A}$ & $1.22 \mathrm{~B}$ \\
\hline Medio & $1.0 \mathrm{C}$ & $1.10 \mathrm{D}$ & $1.36 \mathrm{~A}$ & 1.24 \\
\hline Inferior & $1.0 \mathrm{~B}$ & $1.13 \mathrm{~B}$ & $1.25 \mathrm{~A}$ & $1.11 \mathrm{~B}$ \\
\hline
\end{tabular}

Las muestras de suelo fueron incubadas en recipientes cerrados en una cámara de crecimiento a $24{ }^{\circ} \mathrm{C}$ y a $80 \%$ de capacidad de campo. El $\mathrm{CO}_{2}$ respirado fue atrapado en 0.5 $\mathrm{N}$ de $\mathrm{NaOH}$ y el exceso fue titulado con $0.5 \mathrm{~N}$ de $\mathrm{HCl}$ en presencia de cloruro de bario. $\mathrm{La}$ determinación de $\mathrm{CO}_{2}$ fue realizada a los 2, 7, 14, 27, 36, 44, 52 y 59 días desde el momento de incubación. Cuando la respiración no varió en función del tiempo, se consideró que la tasa era constante, lo que indicaría que la actividad microbiana sería estable.

Biomasa microbiana del suelo, carbono y nitrógeno. Para determinar la biomasa microbiana se tomaron 4 repeticiones por perfil y estrato de profundidad, compuestas por 6 submuestras recolectadas con barreno tubular de $3.2 \mathrm{~cm}$ de diámetro. Los extractos se realizaron de acuerdo con el método de fumigación-extracción con cloroformo adaptado por Voroney et al. (2007) de la versión original de Jenkinson y Powlson (1976). La relación suelo/extractante empleada para los tratamientos fumigados con cloroformo y sin fumigar fue de $30 \mathrm{~g}$ de suelo y $60 \mathrm{~mL}$ de $\mathrm{K}_{2} \mathrm{SO}_{4} 0.5 \mathrm{M}$. En simultáneo se determinó el contenido de humedad. El contenido de carbono orgánico de la biomasa microbiana se determinó por el método propuesto por Vance et al. (1987) y fue calculado como el cociente entre la diferencia de las concentraciones de carbono de las muestras fumigadas y sin fumigar, y un coeficiente de 0.45 propuesto por Vance et al. (1987) y Wu et al. (1990). El contenido de nitrógeno total de la biomasa microbiana fue determinado por el método de digestión de Kjeldahl, de acuerdo con Brookes et al. (1985), y fue calculado de manera similar al carbono, pero utilizando un coeficiente de 0.54, según Brookes et al. (1985).

Cociente metabólico. En la bibliografía existen distintos abordajes a fin de establecer el cálculo del cociente metabólico. Algunos trabajos se refieren a la respiración en las primeras 20 horas (Anderson and Domsch 1990), mientras que otros lo calculan como $\mathrm{mg} \mathrm{C}-\mathrm{CO}_{2} \cdot \mathrm{mg} \mathrm{C}^{-}-\mathrm{BM}^{-1} \cdot \mathrm{d}^{-1}$, considerando la respiración a los 3, 7, 10, 14 y 21 días (Saviozi et al. 2001; Romaniuk et al. 2014). Pero todos los trabajos concuerdan en que en ese lapso, la actividad biológica se estabiliza y la tasa de respiración corresponde a la respiración basal del suelo. El cociente metabólico se calculó mediante la relación entre el carbono liberado 
en la respiración de suelo y el carbono de la biomasa microbiana. Para ello se utilizaron los datos de respiración a tasa constante y se expresaron como tasa de respiración (mg C.d $\mathrm{d}^{-1} . \mathrm{kg}$ suelo $\left.^{-1}\right)$.

Relación hongo/bacteria. A partir del muestreo de biomasa microbiana en cada suelo y estrato de profundidad se separaron $10 \mathrm{~g}$ de suelo y se los congeló a $-20^{\circ} \mathrm{C}$ hasta la extracción de ADN. Para las extracciones de ADN del suelo se utilizó el kit Power Soil $^{\mathrm{TM}}$ (MO BIO Laboratories Inc.) según las especificaciones del producto. Para la cuantificación de bacterias y hongos totales se utilizó un termociclador en tiempo real (qPCR) (Eppendorf Mastercycler). Las curvas standard fueron corridas por triplicado con 5 diluciones de un plásmido que contenía una copia de longitud completa de los genes rRNA16S y $18 S$ de Escherichia coli. El set de primers fue R534 5'-ATTACCGCG GCTGCTGG3' y 338P 5'-C CTACGGGAGGCAGCAG-3' para 16S (Muyzer et al. 1993) y FU18S1 5'GGAAACTCACCAGGTCCAGA-3' y SSU-1536 5'-ATTGCAATGCYCTATCCCCA-3' para 18S (Borneman and Hartin 2000). Las amplificaciones fueron llevadas a cabo para un volumen total de reacción de $25 \mu \mathrm{L}$. La mezcla de reacción para qPCR constó de 10 ng de ADN del suelo, $0.5 \mu \mathrm{L}$ de cada primer, $12.5 \mu \mathrm{L}$ de mezcla real 2x para SYBR Green (Biodynamics) y $0.5 \mu \mathrm{L}$ de BSA $(10 \mu \mathrm{g} / \mathrm{mL})$. Luego de completar la reacción en cadena de la polimerasa, se efectuó el análisis de la curva de melting implementando el software Mastercycler ep realplex (versión 2.2), para determinar la existencia de dímeros de primers u otra fuente de error a un nivel detectable para la medición de fluorescencia con SYBER Green. A partir de estos datos se determinó la relación 18S/16S, que representa la relación hongo/bacteria (Fierer et al. 2005).

Los resultados se analizaron estadísticamente mediante análisis de la varianza, y las diferencias entre medias a través de la prueba de Tukey. Además, se realizaron regresiones lineales y no lineales entre respiración y carbono de la biomasa microbiana en función al carbono total del suelo y entre respiración en función al carbono de la biomasa microbiana y al nitrógeno de la biomasa microbiana. También se realizó un análisis multivariado para obtener una representación gráfica de las variables en el plano de los dos primeros componentes principales. El software utilizado para los análisis fue InfoStat (Di Rienzo et al. 2013).

\section{Resultados}

Los suelos provenientes de vegetación natural presentaron, en el estrato superior del perfil, mayor respiración acumulada que los suelos agrícolas (Figura 2a). Luego de 59 días de incubación, la respiración acumulada de los suelos correspondientes a vegetación natural casi triplicó a la de los suelos agrícolas. En los estratos medio e inferior, la respiración acumulada, fue menor a la del estrato superior, pero con menores diferencias debidas al uso del suelo (Figura 2b y c).

Los contenidos de carbono de la biomasa microbiana del suelo fueron mayores en el estrato superior con respecto a los otros dos estratos, y los suelos provenientes de vegetación natural presentaron valores superiores que los agrícolas (Tabla 2). En el estrato medio del perfil no se detectaron diferencias significativas entre usos del suelo, mientras que en el estrato inferior se presentó un patrón similar al superior, con mayores contenidos en los suelos provenientes de vegetación natural.

Los contenidos de nitrógeno de la biomasa microbiana del suelo también fueron mayores en el estrato superior con respecto a los otros dos estratos (Tabla 2). Los suelos provenientes de vegetación natural también presentaron mayores contenidos que los suelos agrícolas, $\mathrm{y}$ las diferencias se registraron en los tres estratos (Tabla 2).

El cociente metabólicono presentó diferencias estadísticas significativas entre los suelos en los estratos superior y medio. Por su parte, el estrato inferior presentó algunas diferencias entre sitios, que no fueron consistentes con el uso del suelo (Tabla 2).

El uso del suelo tuvo un efecto significativo en la relación hongo/bacteria en el estrato superior, donde los suelos provenientes de vegetación natural tuvieron mayor proporción de hongos con respecto al uso agrícola (Figura 3). Por el contrario, en los estratos medio e inferior del perfil, esta relación no estuvo condicionada por el uso del suelo (Figura 3).

El contenido de carbono explicó 66\% de la variación observada en la respiración $\left(P^{\prime} 0.0001\right)$ en el estrato superior. Sin embargo, la relación entre ambas variables solo fue lineal y positiva en el rango entre $0.44 \mathrm{y}$ $1.56 \%$ de carbono (Figura $4 a$ ), mientras que los incrementos en los contenidos de $\mathrm{C}$ del suelo por encima de dichos valores tendrían 


\section{Respiración (mg $\mathrm{C} \mathrm{kg}^{-1}$ suelo)}

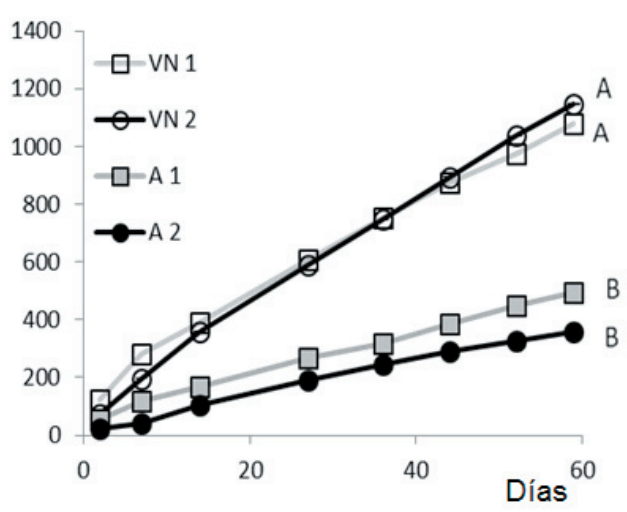

Respiración (mg $\mathrm{C} \mathrm{kg}^{-1}$ suelo)
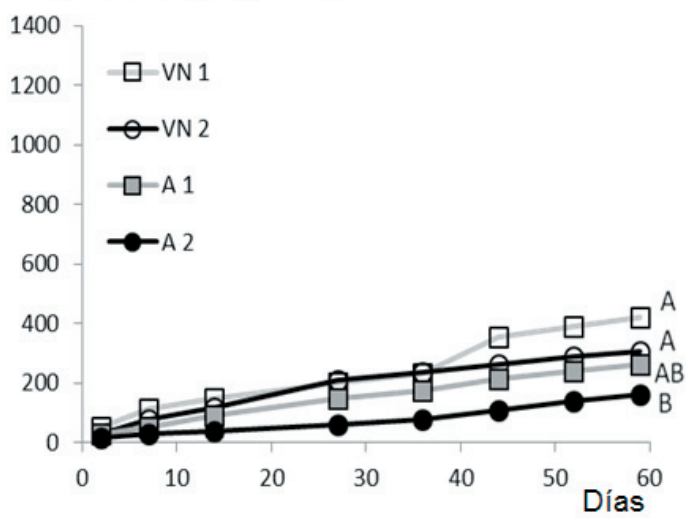

Respiración (mg $\mathrm{C} \mathrm{kg}^{-1}$ suelo)

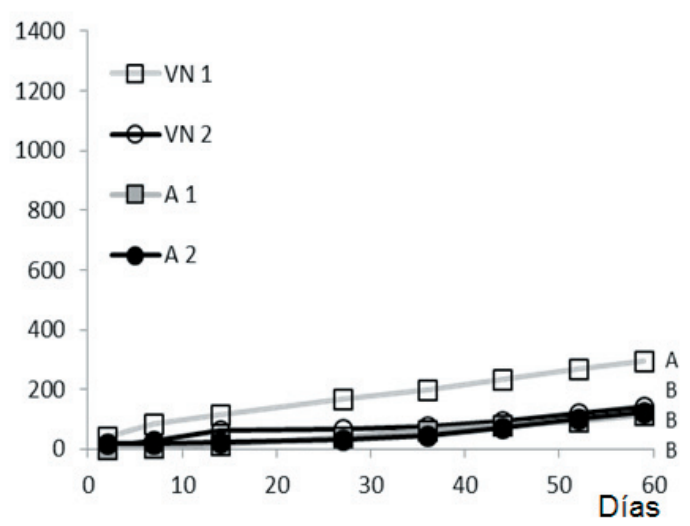

Figura 2. Respiración acumulada en suelos bajo vegetación natural (VN1 y VN2) y agrícola (A1 y A2), en los estratos de profundidad a) superior, b) medio, c) inferior. Letras distintas indican diferencias estadísticas significativas entre uso para el mismo estrato.

Figure 2. Accumulated respiration in soils under natural vegetation (VN1 and VN2) and agriculture (A1 and A2) in upper (a), medium (b), and lower (c) depth layers of the profile. Different letters indicate statistically significant differences between soil uses for the same depth layer.

un efecto menor sobre la respiración de suelo. En los estratos medio e inferior no se detectó una relación significativa entre estas variables

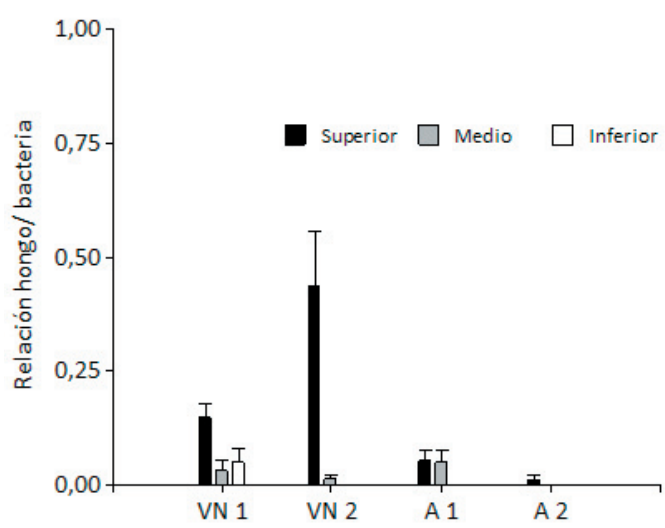

Figura 3. Relación hongo/bacteria en suelos bajo vegetación natural (VN1 y VN2) y agrícola (A1 y A2), en los estratos de profundidad superior, medio e inferior.

Figure 3. Fungi/bacteria ratio in soils under natural vegetation (VN1 and VN2) and agriculture (A1 and A2), in upper (a), medium (b), and lower (c) depth layers of the profile.

a)
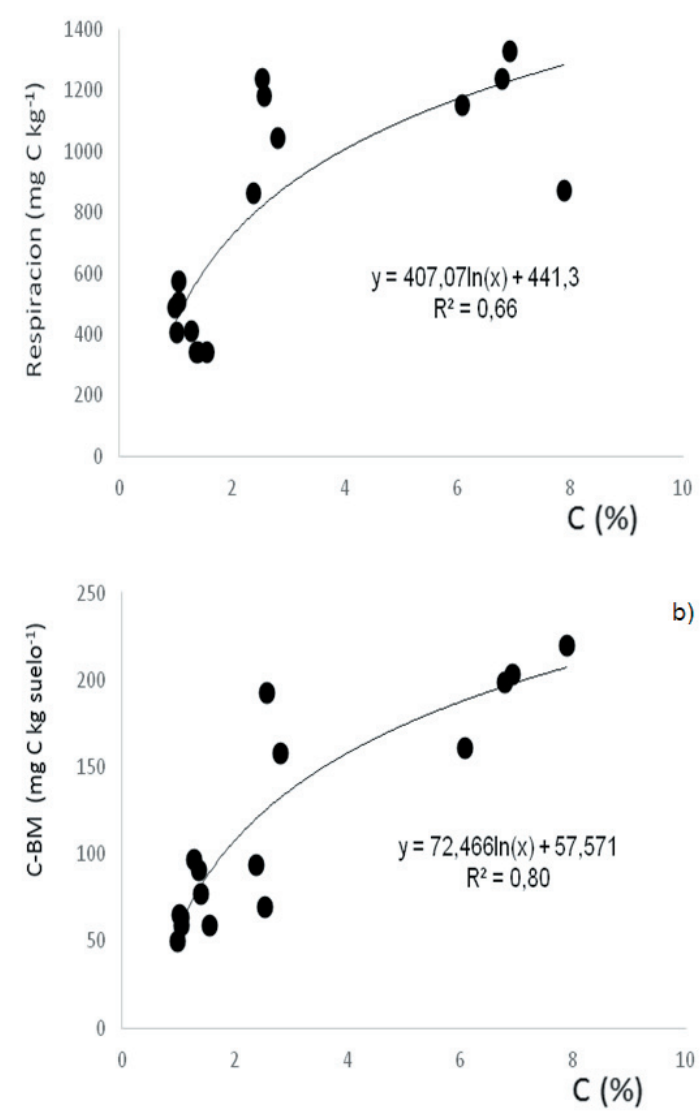

Figura 4. Respiración de suelo (a) y carbono de la biomasa microbiana (C-BM) (b) en función del contenido de carbono (C) en el estrato superior.

Figure 4. Soil respiration (a) and microbial biomass carbon (C-BM) (b) as a function of carbon (C) in upper depth layers of the profile. 
Tabla 2. Carbono y nitrógeno de la biomasa microbiana y cociente metabólico en suelos bajo distintos usos: vegetación natural (VN1 y VN2) y agricultura (A1 y A2). Letras mayúsculas distintas indican diferencias estadísticas significativas entre uso para el mismo estrato. Letras minúsculas distintas indican diferencias estadísticas significativas entre estrato en el mismo uso.

Table 2. Carbon and nitrogen in microbial biomass and metabolic quotient in soils under natural vegetation (VN1 and VN2) and agriculture (A1 and A2). Different uppercase letters indicate statistically significant differences between uses for the same depth layers of the profile. Different lowercase letters indicate statistically significant differences between depth layers of the profile for the same soil.

\begin{tabular}{|c|c|c|c|c|}
\hline & \multicolumn{2}{|c|}{ Vegetación natural } & \multicolumn{2}{|c|}{ Agricultura } \\
\hline & VN1 & VN2 & A1 & A2 \\
\hline & \multicolumn{4}{|c|}{ Carbono de la biomasa microbiana ( $\mu \mathrm{g} / \mathrm{g}$ suelo) } \\
\hline Superior & 128.5 a $\mathrm{AB}$ & 195.5 a A & 81.0 a B & 59.2 a C \\
\hline Medio & $40.1 \mathrm{~b} \mathrm{~A}$ & $47.0 \mathrm{~b} \mathrm{~A}$ & $42.7 \mathrm{ab} \mathrm{A}$ & $26.8 \mathrm{~b} \mathrm{~A}$ \\
\hline \multirow[t]{2}{*}{ Inferior } & $36.9 \mathrm{~b} \mathrm{AB}$ & $40.5 \mathrm{~b} \mathrm{~A}$ & $27.0 \mathrm{~b} \mathrm{BC}$ & $17.5 \mathrm{~b} \mathrm{C}$ \\
\hline & \multicolumn{4}{|c|}{ Nitrógeno de la biomasa microbiana ( $\mu \mathrm{g} / \mathrm{g}$ suelo) } \\
\hline Superior & 36.1 a $A$ & 26.2 a $\mathrm{A}$ & 24.0 a A & 6.4 a B \\
\hline Medio & $13.2 \mathrm{~b} \mathrm{~B}$ & $15.1 \mathrm{~b} \mathrm{~A}$ & $10.0 \mathrm{~b} \mathrm{BC}$ & $3.6 \mathrm{ab} \mathrm{C}$ \\
\hline Inferior & $13.6 \mathrm{~b} \mathrm{~A}$ & $8.3 \mathrm{~b} \mathrm{AB}$ & $6.7 \mathrm{~b} \mathrm{~B}$ & $3.7 \mathrm{~b} \mathrm{C}$ \\
\hline \multicolumn{5}{|c|}{ Cociente metabólico (g C.mg C-BM ${ }^{-1} \cdot \mathrm{d}^{-1}$ ) } \\
\hline Superior & 0.16 a $\mathrm{A}$ & 0.11 a A & 0.10 a $\mathrm{A}$ & 0.10 a $\mathrm{A}$ \\
\hline Medio & 0.15 a A & 0.11 a A & 0.10 a A & 0.12 a $\mathrm{A}$ \\
\hline Inferior & 0.14 a A & $0.05 \mathrm{~b} \mathrm{C}$ & 0.07 a BC & $0.12 \mathrm{a} A B$ \\
\hline
\end{tabular}

$\left(R^{2}=0.01\right.$ y 0.03 , respectivamente). La biomasa microbiana se relacionó de forma positiva con los contenidos de carbono de los suelos en estrato superior (Figura $4 \mathrm{~b})\left(P^{\prime} 0.0001 ; \mathrm{R}^{2}=0.80\right)$, mientras que los datos correspondientes a los estratos medio e inferior no ajustaron a ningún tipo de ecuación $\left(\mathrm{R}^{2}=0.03\right.$ y 0.04 , respectivamente).

A fin de establecer los valores umbrales para los indicadores biológicos evaluados, se recurrió a un estudio previo de la región semiárida pampeana en el que se evaluó la incidencia de los contenidos de la materia orgánica sobre indicadores físicos y químicos del suelo. Ese trabajo arrojó $\sim 3 \%$ como valor umbral preliminar del contenido de materia orgánica (equivalente a 1.74\% de carbono) (Fernández et al. 2016). En este sentido, al reemplazar en la ecuación logarítmica que relaciona la respiración de los microorganismos del suelo en función del contenido de carbono (Figura 4a) se puede inferir un posible valor umbral de respiración de $666.7 \mathrm{mg} \mathrm{C} / \mathrm{kg}$ suelo. Del mismo modo, y también considerando $1.74 \%$ de carbono en la ecuación que relaciona el carbono de la biomasa microbiana con el carbono del suelo (Figura $4 b$ ), se obtuvo un posible valor umbral del carbono de la biomasa microbiana correspondiente a $97.7 \mu \mathrm{g} / \mathrm{g}$ suelo.

Tanto la actividad biológica inferida a partir de la respiración como la abundancia de microorganismos, reflejada en la cuantificación de la biomasa microbiana, constituyen
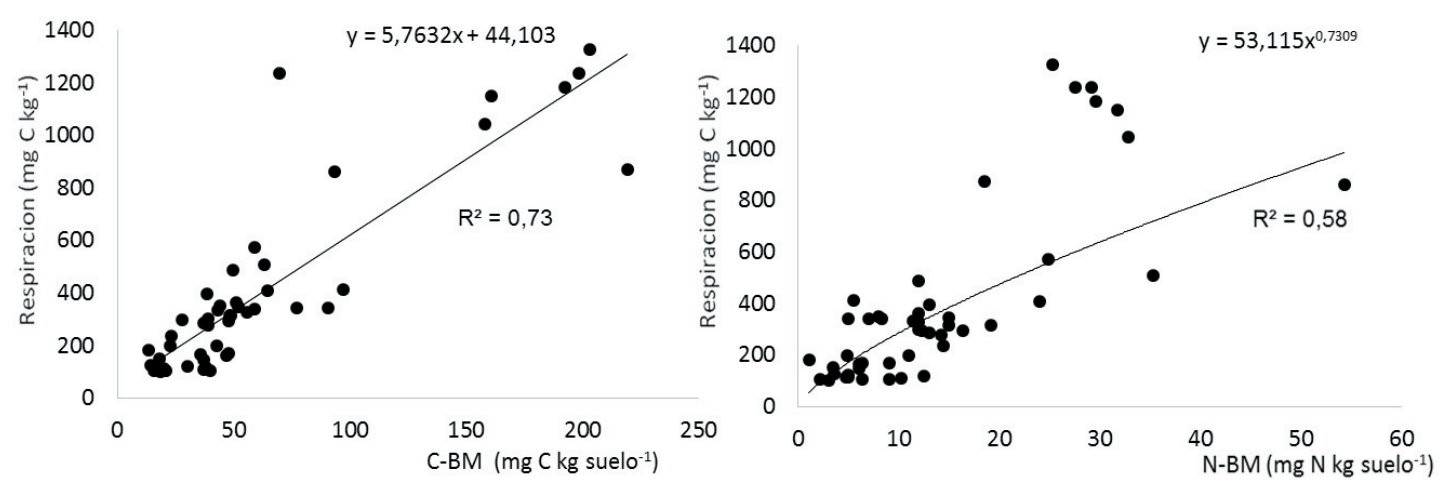

Figura 5. Respiración de suelo en función de: a) carbono de la biomasa microbiana (C-BM) y b) nitrógeno de la biomasa microbiana (N-BM).

Figure 5. Soil respiration in relation to: a) microbial biomass carbon (C-BM) and b) microbial biomass nitrogen (N$\mathrm{BM})$. 


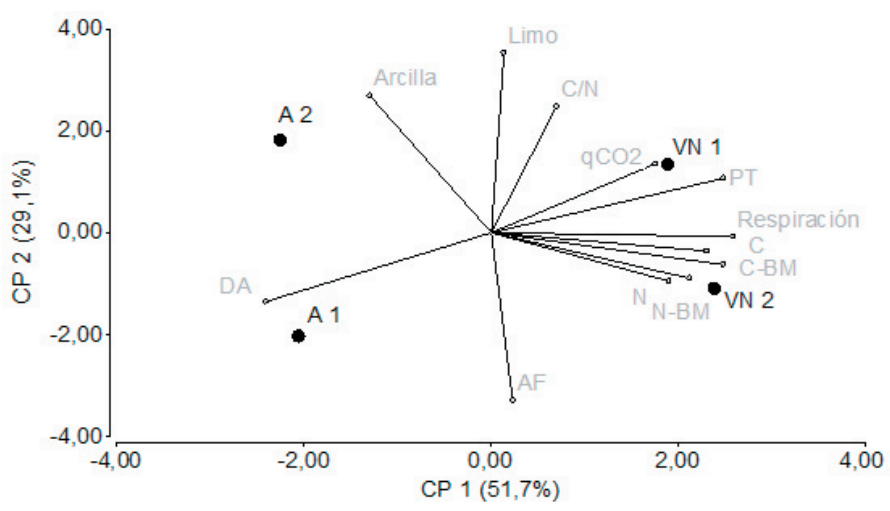

Figura 6. Análisis de componentes principales en suelos bajo uso agrícola (A1 y A2) y con vegetación natural (VN1 y $\mathrm{VN2}$ ). Las variables fueron arena fina (AF), carbono $(\mathrm{C})$, nitrógeno $(\mathrm{N})$, relación carbono nitrógeno $(\mathrm{C} / \mathrm{N})$, cociente metabólico $\left(\mathrm{qCO}_{2}\right)$, porosidad total $(\mathrm{PT})$, carbono de la biomasa microbiana (C-BM), nitrógeno de la biomasa microbiana (N-BM).

Figure 6. Principal components analysis for agricultural (A1 and A2) and natural vegetation (VN1 and VN2) soils. Variables were fine sand $(\mathrm{AF})$, carbon $(\mathrm{C})$, nitrogen, carbon/nitrogen ratio $(\mathrm{C} / \mathrm{N})$, metabolic quotient $\left(\mathrm{qCO}_{2}\right)$, total porosity $(\mathrm{PT})$, microbial biomass carbon (C-BM) and nitrogen (N-BM). variables acopladas entre sí que responden a cambios en el corto plazo. La respiración de los microorganismos se asoció en 73\% ( $\left.P^{\prime} 0.0001\right)$ con el carbono de la biomasa microbiana, lo que dio como resultado una regresión lineal y positiva entre ambas variables (Figura 5a). En menor grado se asoció la respiración con el nitrógeno de la biomasa microbiana (58\%, $\left.P^{\prime} 0.0001\right)$, aunque la regresión obtenida entre estas variables fue potencial (Figura 5b).

El análisis de componentes principales mostró que los suelos agrícolas se ubicaron en el sector definido por la densidad aparente, mientras que los suelos provenientes de vegetación natural se ubicaron en el sector definido por el carbono, el cociente metabólico, la porosidad total y el carbono y el nitrógeno de la biomasa microbiana (Figura 6). El suelo A2 se diferenció por poseer un mayor contenido de arcilla que el A1, el cual, por su parte, presentó mayores contenidos de arena fina. VN1 se asoció mayormente al cociente metabólico, mientras que VN2 estuvo más relacionado con el carbono y el nitrógeno de la biomasa microbiana.

\section{DisCUSIÓN}

Los resultados demostraron que el uso del suelo incidió sobre la actividad biológica, con valores mayores de respiración, carbono y nitrógeno de la biomasa microbiana y relación hongo/bacteria en los suelos de vegetación natural con respecto a los suelos agrícolas. Las mayores diferencias siempre se hallaron en el estrato superior del suelo, mientras que a mayor profundidad, en muchos casos no se hallaron diferencias significativas. Este patrón vertical también se observó en comparaciones entre sistemas de labranza, en los que no se constataron diferencias en profundidades mayores que $10 \mathrm{~cm}$ (Spedding et al. 2004).
Se consideraron como los valores de línea de base de indicadores biológicos para suelos franco y franco arenoso de la región semiárida pampeana aquellos que corresponden al uso con vegetación natural. Los resultados de los indicadores muestran una diferencia amplia entre el valor de línea de base y el de los suelos agrícolas. El valor de línea de base de la respiración acumulada fue $1146 \mathrm{mg} \mathrm{C} / \mathrm{kg}$ suelo, el uso en VN1 presentó un valor cercano al de la línea de base (1081 mg C/kg suelo), pero bajo el uso agrícola, los datos fueron muy inferiores y correspondieron a 493 y $359 \mathrm{mg}$ $\mathrm{C} / \mathrm{kg}$ suelo para $\mathrm{A} 1$ y A2, respectivamente. El valor umbral de $667 \mathrm{mg} \mathrm{C} / \mathrm{kg}$ suelo hallado para los suelos con $1.74 \%$ de C se encontró más próximo al valor promedio de suelos agrícolas que al valor de línea de base que se propone en este estudio. Estos resultados se corresponden con los reportados por la bibliografía: en suelos con vegetación natural, la respiración podría llegar a ser 2 ó 3 veces mayor con respecto a suelos cultivados, asociado al aumento de la actividad microbiana del suelo a partir de los exudados radicales de las herbáceas nativas (Meier et al. 2017). Estudios desarrollados por Fernández et al. (2010) y Noellemeyer et al. (2008) en suelos franco de la región semiárida, con contenidos de carbono de $1.5 \%$, encontraron que la respiración acumulada fue similar cuando se compararon diferentes usos de suelo, pero con pocos años de efectos acumulados. En ambos estudios, el valor de respiración encontrado fue similar al indicado como umbral del presente trabajo, distando del valor de línea de base que se propone. Pero resulta difícil comparar valores de línea de base y umbrales con respecto a este indicador debido a las diferentes metodologías que se usan para determinar la respiración. Además, el muestreo que se empleó también es diverso; muchos trabajos reportan valores de respiración en tamaños de agregados (Jiang et al. 2011), en suelos enteros tamizados por 2 
$\mathrm{mm}$ y en cilindros sin disturbar (Noellemeyer et al. 2008).

El carbono de la biomasa microbiana también presentó una diferencia amplia entre el valor de línea de base y el de los suelos agrícolas. El valor de línea de base fue 195 $\mu \mathrm{g} / \mathrm{g}$ suelo, que fue muy superior respecto al del uso agrícola, en el que se midió 81 y 59 $\mu \mathrm{g} / \mathrm{g}$ suelo para A1 y A2, respectivamente. De la misma manera que lo encontrado para la respiración, el valor umbral de $97.7 \mu \mathrm{g} / \mathrm{g}$ suelo estuvo más próximo al valor de los suelos agrícolas que al valor de línea de base que se propone en este estudio. En la literatura se informan valores mayores que los encontrados en este estudio en los contenidos de carbono de la biomasa microbiana del suelo entre sistemas de labranza, rotaciones de cultivos y usos del suelo que varían entre 250 a 600 $\mu \mathrm{g} / \mathrm{g}$ suelo para regiones frías y tropicales, respectivamente (Ekenler and Tabatabai 2003; Frasier et al. 2016a), aunque algunos estudios recientes en pasturas perennes de Alemania arrojaron valores inferiores (entre 114 y 126 $\mu \mathrm{g} / \mathrm{g}$ suelo) al propuesto como línea de base (Blagodatskaya et al. 2011), mientras que en un pastizal natural semiárido de Australia se hallaron valores en un rango de 700 a 900 $\mu \mathrm{g} / \mathrm{g}$ suelo (Canarini et al. 2017). McDaniel y Grandy (2016), en suelos agrícolas de textura franca de Iowa (EEUU), obtuvieron un rango entre 60 y $1661 \mu \mathrm{g} / \mathrm{g}$ suelo en todas las estaciones y en diferentes rotaciones de cultivos. El mayor valor del rango hallado se encontró muy por encima con respecto a los datos presentados en nuestro trabajo. Aunque Frasier et al. (2016a) informaron para la región semiárida pampeana un rango (entre 143 y 295 $\mu \mathrm{g} / \mathrm{g}$ suelo) que contiene el valor de línea de base que se propone en este estudio; ellos atribuyen estos menores valores al clima de la pampa semiárida. Hasta la fecha no existen para esta región estudios de campo en los que se haya monitoreado el carbono de la biomasa microbiana en sistemas de manejo de suelos durante un período más largo.

El cociente metabólico no permitió establecer una relación con los contenidos de carbono de los suelos y, por lo tanto, no se pudo establecer el valor umbral. El valor de línea de base, obtenido en los suelos con vegetación natural (0.11), fue similar al de los suelos agrícolas (0.10). Estos datos son comparables con el valor reportado para suelos bajo agricultura en Iowa, EEUU (McDaniel and Grandy 2016). En cambio, Moscatelli et al. (2007) obtuvieron menores valores en suelos bajo pasturas de gramíneas que bajo agricultura convencional. Sin embargo, bajo las condiciones de nuestro estudio, el escaso rango entre los suelos bajo vegetación natural y agricultura sugiere que este indicador no sería sensible para detectar diferencias de acuerdo al uso del suelo dentro de los sistemas agrícolas, tal como ya lo plantearon Wardle y Ghani (1995) y confirmaron Zuber y Villamil (2016), quienes encontraron valores similares en suelos bajo siembra directa y labranza convencional.

El uso del suelo incidió en la relación hongo/ bacteria, y se comprobó una relación mayor en suelos bajo vegetación natural. Esto significó que en los suelos agrícolas, la participación de hongos en la biomasa microbiana fue considerablemente menor que en los suelos bajo vegetación natural. El efecto negativo de las labranzas sobre la diversidad de la microbiota del suelo (Coudrain et al. 2016), y especialmente sobre la población de hongos, también fue observado por Sun et al. (2011), quienes hallaron mayor dominancia de bacterias en siembra directa comparado con labranza convencional. En sistemas de pastizales o pasturas cultivadas con gramíneas y leguminosas se documentaron valores de relación hongo/bacteria entre 0.4-0.3 (de Vries et al. 2006), similares a lo hallado en los suelos bajo vegetación natural. En cambio, los valores para suelos bajo agricultura convencional son inferiores a 0.2 (Sun et al. 2011).

La diferencia reflejada entre el valor línea de base de los suelos provenientes de vegetación natural y el de los suelos agrícolas representó una pérdida de la biodiversidad como consecuencia del uso agrícola, que fue documentada recientemente en trabajos realizados en diferentes regiones (French et al. 2017). De acuerdo con los resultados, y sobre la base de evidencia reciente, la biomasa microbiana de los suelos de la región semiárida pampeana estaría dominada por bacterias (Frasier et al. 2016a), con una proporción menor a $20 \%$ de hongos. En pastizales de Europa se halló una similar predominancia de bacterias (de Vries et al. 2006). La conversión de pastizales a cultivos agrícolas anuales produce cambios en la estructura de la comunidad microbiana del suelo, aunque generalmente no causa una pérdida de la diversidad de la población bacteriana o la disminución de determinadas poblaciones benéficas (e.g., fijadores de N), sino que se reduce la diversidad de los hongos, tal como se demostró en un estudio extensivo en Inglaterra (French et al. 2017). Se ha postulado que los 
hongos serían más eficientes en secuestrar el C debido a su mayor eficiencia (Kramer et al. 2012), y que tendrían una tasa de ciclado de los elementos más lenta que las bacterias (Paul et al. 2015). En este sentido, se infiere que el uso bajo vegetación natural ofreció un ambiente con mayor contenido de carbono y nitrógeno en el suelo con respecto al uso agrícola, lo cual favoreció poblaciones más eficientes y tolerantes al estrés abiótico como los hongos y que estas condiciones derivaron en mayores valores de cociente metabólico. Lo expuesto coincide con Kong et al. (2011) y Frasier et al. (2016b), quienes demostraron que una mayor actividad biológica no necesariamente conlleva a pérdidas de carbono de la materia orgánica, como muchos autores supusieron con anterioridad (Janzen 2006), sino que altas tasas de ciclado de carbono y nitrógeno se corresponden con altas proporciones de secuestro de estos elementos. La mayor actividad biológica en la vegetación natural reflejada con valores más elevados del cociente metabólico permitiría inferir que la abundancia de sustrato en estos suelos favorecería el secuestro de carbono, a pesar de una posible baja eficiencia del uso de carbono por parte de los microorganismos. Los suelos agrícolas resultaron degradados en su calidad biológica a pesar de la ambigüedad del valor del cociente metabólico. Menores valores de carbono en suelos agrícolas dieron lugar a la reducción en la actividad biológica reflejada por la respiración del suelo, al tamaño del pool biológico representado por la biomasa microbiana y a la diversidad tal como lo indicaría la relación hongo/bacteria.

Los datos permitieron establecer valores de línea de base y umbrales para la respiración acumulada y el carbono de la biomasa microbiana. Los valores umbrales encontrados constituyen una aproximación preliminar para los suelos franco y franco arenosos de la región semiárida pampeana que requerirá de un estudio más específico para establecer con mayor precisión dicho valor. El cociente metabólico no resultó ser un indicador sensible, y por no encontrarse una relación significativa con los contenidos de carbono del suelo no fue posible establecer un valor umbral para este parámetro.

Agradecimientos. El trabajo se llevó a cabo gracias a la Beca de Formación de INTA y a Proyectos Regional (1282101) y Nacional (Caracterización y funcionalidad de la biota del suelo PN SUELOS-PE 1134043) de INTA.

\section{REFERENCIAS}

Anderson, T. 2003. Microbial eco-physiological indicators to assess soil quality. Agriculture, Ecosystems and Environment 98:285-293.

Anderson, T., and K. Domsch. 1990. Application of eco-physiological quotients (qCO2 and qD) on microbial biomasses from soils of different cropping histories. Soil Biology and Biochemistry 22:251-255.

Anderson, T., and K. Domsch. 1993. The metabolic quotient for CO2 (qCO2) as a specific activity parameter to assess the effects of environmental conditions, such as $\mathrm{pH}$, on the microbial biomass of forest soils. Soil Biology and Biochemistry 25:393-395.

Ashman, M. 2003. Are the links between soil aggregate size class, soil organic matter and respiration rate artefacts of the fractionation procedure? Soil Biology and Biochemistry 35:435-444.

Ashman, M., P. Hallett, P. Brookes and J. Allen. 2009. Evaluating soil stabilisation by biological processes using stepwise aggregate fractionation. Soil Till Res 102:209-215.

Benayas, J., P. Barral, and P. Meli. 2017. Lecciones de cuatro meta-análisis globales sobre la restauración de la biodiversidad y los servicios ecosistémicos. Ecología Austral 27:193-198.

Blagodatskaya E., T. Yuyukina, S. Blagodatsky, and Y. Kuzyakov. 2011. Turnover of soil organic matter and of microbial biomass under C3-C4 vegetation change: Consideration of $13 \mathrm{C}$ fractionation and preferential substrate utilization. Soil Biol Biochem 43:159-166.

Bockstaller, C., P. Girardin, and H. van der Werf. 1997. Use of agro-ecological indicators for the evaluation of farming systems. European Journal of Agronomy 7:261-270.

Borneman, J., and R. Hartin. 2000. PCR primers that amplify fungal rRNA genes from environmental samples. Applied and Environmental Microbiology 66:4356-60.

Bossio, D., M. Girvan, L. Verchot, J. Bullimore, T. Borelli, A. Albrecht, K. Scow, A. Ball, J. Pretty, and A. Osborn. 2005. Soil microbial community response to land use change in an agricultural landscape of western Kenya. Microbial Ecology 49:50-62.

Brookes, P., A. Landman, G. Pruden, D. Jenkinson, and R. Station. 1985. Chloroform fumigation and the release of soil nitrogen: A rapid direct extraction method to measure microbial biomass nitrogen in soil. Soil Biology and Biochemistry 17:837-842.

Canarini, A., P. Mariotte, L. Ingram, A. Merchant, and F. Dijkstra. 2017. Mineral-Associated Soil Carbon is Resistant to Drought but Sensitive to Legumes and Microbial Biomass in an Australian Grassland. Ecosystems 21(2):349-359. doi:10.1007/s10021-017-0152-x 
Costantini, A., D. Cosentino, and A. Segat. 1996. Influence of tillage systems on biological properties of a typic argiudoll soil under continuous maize in central argentina. Soil Till Res 38:265-271.

Cotrufo, M., M. Wallenstein, C. Boot, K. Denef, and E. Paul. 2013. The Microbial Efficiency-Matrix Stabilization (MEMS) framework integrates plant litter decomposition with soil organic matter stabilization: do labile plant inputs form stable soil organic matter? Global Change Biology 19:988-95.

Coudrain, V., M. Hedde, M. Chauvat, P. Maron, E. Bourgeois, B Mary, J. Léonard, F. Ekelund, C. Villenave, and S. Recous. 2016. Temporal differentiation of soil communities in response to arable crop management strategies. Agriculture, Ecosystems and Environment 225:12-21.

De Vries, F., E. Hoffland, N. van Eekeren, L. Brussaard, and J. Bloem. 2006. Fungal/bacterial ratios in grasslands with contrasting nitrogen management. Soil Biology and Biochemistry 38:2092-2103.

Di Rienzo, J., F. Casanoves, M. Balzarini, L. Gonzalez, M. Tablada, and C. Robledo. 2013. Infostat - Sofware estadístico. Universidad Nacional de Córdoba, Argentina [WWW Document]. Universidad Nacional de Córdoba, Argentina.

Ekenler, M., and M. Tabatabai. 2003. Effects of liming and tillage systems on microbial biomass and glycosidases in soils. Biology and Fertility of Soils 39:51-61.

Fernández R., A. Quiroga, C. Álvarez, C. Lobartini, and E. Noellemeyer. 2016. Valores umbrales de algunos indicadores de calidad de suelos en molisoles de la región semiárida pampeana. Rev Ciencia del Suelo 34:279-292.

Fernández, R., A. Quiroga, C. Zorati, and E. Noellemeyer. 2010. Carbon contents and respiration rates of aggregate size fractions under no-till and conventional tillage. Soil Till Res 109:103-109.

Fierer, N., J. Jackson, R. Vilgalys, and R. Jackson. 2005. Assessment of Soil Microbial Community Structure by Use of Taxon-Specific Quantitative PCR Assays 71:4117-4120.

Figuerola, E., L. Guerrero, S. Rosa, L. Simonetti, M. Duval, J. Galantini, J. Bedano, L. Wall, and L. Erijman. 2012. Bacterial indicator of agricultural management for soil under no-till crop production. PloS One 7:1-12.

Frasier, I., E. Noellemeyer, E. Figuerola, L. Erijman, H. Permingeat, and A. Quiroga. 2016a. High quality residues from cover crops favor changes in microbial community and enhance $\mathrm{C}$ and $\mathrm{N}$ sequestration. Global Ecology and Conservation 6:242-256.

Frasier, I., A. Quiroga, and E. Noellemeyer. 2016b. Effect of different cover crops on C and N cycling in sorghum NT systems. Science of The Total Environment 562:628-639.

French, K., A. Tkacz, and L. Turnbull. 2017. Conversion of grassland to arable decreases microbial diversity and alters community composition. Applied Soil Ecology 110:43-52.

Geyer, K., E. Kyker-Snowman, A. Grandy, and S. Frey. 2016. Microbial carbon use efficiency: accounting for population, community, and ecosystem-scale controls over the fate of metabolized organic matter. Biogeochemistry 127:173188.

Griffiths, R., B. Thomson, P. Plassart, H. Gweon, D. Stone, R. Creamer, P. Lemanceau, and M. Bailey. 2016. Mapping and validating predictions of soil bacterial biodiversity using European and national scale datasets. Applied Soil Ecology 97:61-68.

Janzen, H. 2006. The soil carbon dilemma: Shall we hoard it or use it? Soil Biology and Biochemistry 38:419-424.

Jenkinson, D., and D. Powlson. 1976. The effects of biocidal treatments on metabolism in soil-I. Fumigation with chloroform. Soil Biology and Biochemistry 8:167-177.

Jiang X., A. Wright, J. Wang, and Z. Li. 2011. Long-term tillage effects on the distribution patterns of microbial biomass and activities within soil aggregates. CATENA 87:276-280.

Kallenbach, C. M., S. D. Frey, A. S. Grandy, J. Lehmann, et al. 2016. Direct evidence for microbial-derived soil organic matter formation and its ecophysiological controls. Nature Communications 7:13630.

Kong, A., K. Scow, A. Córdova-Kreylos, W. Holmes, and J. Six. 2011. Microbial community composition and carbon cycling within soil microenvironments of conventional, low-input, and organic cropping systems. Soil Biology and Biochemistry 43:20-30.

Kramer, S., S. Marhan, L. Ruess, W. Armbruster, O. Butenschoen, H. Haslwimmer, Y. Kuzyakov, J. Pausch, N. Scheunemann, J. Schoene, A. Schmalwasser, K. Totsche, F. Walker, S. Scheu, and E. Kandeler. 2012. Carbon flow into microbial and fungal biomass as a basis for the belowground food web of agroecosystems. Pedobiologia 55: 111-119.

Kravchenko, A., W. Negassa, A. Guber, B. Hildebrandt, T. Marsh, and M. Rivers. 2014. Intra-aggregate Pore Structure Influences Phylogenetic Composition of Bacterial Community in Macroaggregates. Soil Science Society of America Journal 78:1924.

McDaniel M., and A. Grandy. 2016. Soil microbial biomass and function are altered by 12 years of crop rotation. SOIL 2:583-599.

Meier, I., A. Finzi, and R. Phillips. 2017. Root exudates increase N availability by stimulating microbial turnover of fast-cycling N pools. Soil Biology and Biochemistry 106:119-128.

Micó, C., M. Peris , L. Recatalá, and J. Sánchez. 2007. Baseline values for heavy metals in agricultural soils in an European Mediterranean region. Science of The Total Environment 378(1-2):13-17.

Moscatelli, M., A. Di Tizio, S. Marinari, and S. Grego. 2007. Microbial indicators related to soil carbon in Mediterranean land use systems. Soil Till Res 97:51-59.

Muyzer, G., E. de Waal, and A. Uitterlinden. 1993. Profiling of complex microbial populations by denaturing gradient gel electrophoresis analysis of polymerase chain reaction-amplified genes coding for $16 \mathrm{~S}$ rRNA. Applied and Environmental Microbiology 59:695-700. 
Nannipieri, P. 1994. The potential use of soil enzymes as indicators of productivity, sustainability and pollution. Pp. 238-244 en C.E. Pankhurst, B. M. Doube, V. V. S. R. Gupta and P. R. Grace (eds.). Soil Biota: Management in Sustainable Farming Systems. CSIRO, Australia.

Negassa, W., A. Guber, A. Kravchenko, T. Marsh, B. Hildebrandt, and M. Rivers. 2015. Properties of Soil Pore Space Regulate Pathways of Plant Residue Decomposition and Community Structure of Associated Bacteria. Plos One 10: $1-22$.

Noellemeyer, E., F. Frank, C. Álvarez, G. Morazzo, and A. Quiroga. 2008. Carbon contents and aggregation related to soil physical and biological properties under a land-use sequence in the semiarid region of central Argentina. Soil Till Res 99:179-190.

Odum, E. 1969. The strategy of ecosystem development. Science 164(3877):262-270.

Pascaud, A., M. Soulas, S. Amellal, and G. Soulas. 2012. An integrated analytical approach for assessing the biological status of the soil microbial community. European Journal of Soil Biology 49:98-106.

Philippot, L., K. Ritz, P. Pandard, S. Hallin, and F. Martin-Laurent. 2012. Standardisation of methods in soil microbiology: progress and challenges. FEMS Microbiology Ecology 82:1-10.

Paul, E., S. Frey, K. Killham, J. Prosser, et al. 2015. Soil Microbiology, Ecology and Biochemistry, Soil Microbiology, Ecology and Biochemistry. 4th ed. Elsevier Inc. Pp. 573-582.

Ponge, J. 2015. The soil as an ecosystem. Biology and Fertility of Soils 51:645-648.

Romaniuk, R., A. Costantini, L. Giuffré, and P. Nannipieri. 2016. Catabolic response and phospholipid fatty acid profiles as microbial tools to assess soil functioning. Soil Use and Management 32:603-612. doi:10.1111/sum.12293.

Romaniuk, R., L. Giuffré, A. Costantini, N. Bartoloni, and P. Nannipieri. 2014. A comparison of indexing methods to evaluate quality of horticultural soils. Part II. Sensitivity of soil microbiological indicators. Soil Research 52:409418.

Saviozzi, A., R. Cardelli, and R. Riffaldi. 2001. A comparison of soil quality in adjacent cultivated, forest and native grassland soils. Plant and Soil 233(2):251-259.

Schloter, M. 2003. Indicators for evaluating soil quality. Agriculture, Ecosystems and Environment 98:255-262.

Segnestam, L. 2002. Indicators of Environmental and Sustainable Development. Theories and Practical Experience, Environmental Economic Series, Paper No 89. Pp. 61. World Bank, Washington DC.

Spedding, T., C. Hamel, G. Mehuys, and C. Madramootoo. 2004. Soil microbial dynamics in maize-growing soil under different tillage and residue management systems. Soil Biology and Biochemistry 36:499-512.

Strickland, M., and J. Rousk. 2010. Considering fungal:bacterial dominance in soils - Methods, controls, and ecosystem implications. Soil Biology and Biochemistry 42:1385-1395.

Sun, B., P. Hallett, S. Caul, T. Daniell, and D. Hopkins. 2011. Distribution of soil carbon and microbial biomass in arable soils under different tillage regimes. Plant and Soil 338:17-25.

Thackway, R., and A. Specht. 2015. Synthesising the effects of land use on natural and managed landscapes. Science of The Total Environment 526:136-152.

Van Bruggen, A., and A. Semenov. 2000. In search of biological indicators for soil health and disease suppression. Applied Soil Ecology 15:13-24.

Vance, E., P. Brookes, and D. Jenkinson. 1987. An extraction method for measuring soil microbial biomass C. Soil Biology and Biochemistry 19:703-707.

Voroney, R., P. Brookes, and R. Beyaert. 2007. Soil microbial biomass C, N, P, and S. Pp. 637-651 en M. R. Carter and E. G. Gregorich (eds.). Soil Sampling and Methods of Analysis. Canadian Society of Soil Science. CRC Press.

Wang, G., and W. Post. 2012. A theoretical reassessment of microbial maintenance and implications for microbial ecology modeling. FEMS Microbiology Ecology 81:610-7.

Wardle, D., and A. Ghani. 1995. A critique of the microbial metabolic quotient $\left(\mathrm{qCO}_{2}\right)$ as a bioindicator of disturbance and ecosystem development. Soil Biology and Biochemistry 27:1601-1610.

Wu, J., R. Joergensen, B. Pommerening, R. Chaussod, and P. Brookes. 1990. Measurement of soil microbial biomass C by fumigation-extraction - an automated procedure. Soil Biology and Biochemistry 22:1167-1169.

Zuber, S., and M. Villamil. 2016. Meta-analysis approach to assess effect of tillage on microbial biomass and enzyme activities. Soil Biol Biochem 97:176-187. 